\title{
El tiempo subjetivo como producto de la doctrina kantiana de la auto-afección
}

\section{The subjective time as a result of the Kantian doctrine of self- affection}

\author{
Matías Orono \\ matiasoro@gmail.com \\ (Universidad de Buenos Aires, Buenos Aires, Argentina)
}

Resumen: El objetivo de este trabajo es demostrar que la doctrina kantiana de la auto-afección da lugar a la consciencia de un tiempo meramente subjetivo. En primer lugar, analizaremos los $\S \S 8$ y 24 de la Crítica de la Razón Pura con el fin de comprender la distinción entre un nivel a priori y otro empírico de auto-afección. En segundo lugar, compararemos la doctrina de la auto-afección y el esquematismo trascendental, con el fin de señalar que la temporalidad a priori de la auto-afección no se identifica con el tiempo objetivo del esquematismo.

Palabras clave: Kant, auto-afección, tiempo subjetivo, esquematismo, tiempo objetivo.
Abstract: The aim of this work is to demonstrate that Kant's doctrine of self-affection leads to a consciousness of a purely subjective time. First, we analyze the $\S \S 8$ and 24 of the Critique of Pure Reason in order to understand the distinction between an a priori and an empirical point of view about self-affection. Second, we compare the doctrine of self-affection with the transcendental schematism to underline that the a priori temporality of selfaffection is not identified with the objective time of the schematism.

Keywords: Kant, self-affection, subjective time, schematism, objective time.

DOI: http://dx.doi.org/10.11606/issn.2318-9800.v19i1p149-168

\section{Introducción}

En este trabajo intentaré señalar que la doctrina kantiana de la auto-afección se halla ligada a un modo de temporalidad meramente subjetiva que no coincide necesariamente con la temporalidad objetiva. El tiempo subjetivo puede ser analizado como un producto de la auto-afección, en el sentido de que sólo podemos ser conscientes de 
la temporalidad subjetiva gracias al acto de auto-afección. Es decir, debemos suponer un tiempo que le pertenece originariamente a la sensibilidad, pero sólo podemos tomar consciencia de él a través de este desdoblamiento del sujeto mediante el cual somos afectados por nosotros mismos. Una de las consecuencias de la tesis aquí presentada consiste en lo siguiente: si bien el tiempo es una intuición que le pertenece originariamente a la sensibilidad, sólo podemos tomar consciencia de ésta, mediante un influjo del entendimiento sobre la sensibilidad - el cual es pensado por Kant como un acto de auto-afección- Asimismo, dado que esta temporalidad ligada a la auto-afección no coincide necesariamente con las determinaciones objetivas del tiempo, sería posible dar cuenta al interior de la gnoseología kantiana, no sólo de la temporalidad de las representaciones vivenciadas de modo intersubjetivo, sino también de aquellas que se reducen al flujo de consciencia meramente individual — tal como pueden ser las alucinaciones o los sueños-. Esta última es una consecuencia que se desprende de nuestra tesis central, aunque por motivos de extensión no será analizada en este trabajo.

En primer lugar, revisaré la doctrina de la auto-afección tal como aparece en los $\S \S 8$ y 24 de la Crítica de la razón pura ${ }^{1}$, señalando que la misma se despliega en dos niveles: uno a priori y otro empírico. Luego, realizaré una breve comparación entre la doctrina de la auto-afección y el esquematismo trascendental, con el fin de señalar los distintos modos de temporalidad implicados en cada uno de estos textos. A mi modo de ver, mientras que la doctrina de la auto-afección da lugar a un tiempo meramente subjetivo, el esquematismo trascendental constituye las determinaciones objetivas del tiempo.

\section{La auto-afección en el $\S 8$ de la Crítica de la razón pura:}

En el marco de unas observaciones destinadas a subrayar los resultados principales de la "Estética trascendental", Kant sostiene que el sentido externo no nos brinda ningún tipo de conocimiento referido a las cosas en sí mismas. Estas observaciones en torno al carácter fenoménico no se limitan sólo a nuestro sentido externo, sino que

1. Los textos citados corresponden a la siguiente traducción al español: KANT, I. Crítica de la razón pura. Traducción de Mario Caimi. Buenos Aires: Colihue, 2007. 
también son válidas para nuestro sentido interno ${ }^{2}$. Por un lado, el contenido empírico o material de nuestro sentido interno depende de las representaciones que nos otorga el sentido externo. Este último sólo nos brinda un conocimiento relacional, sin acceder a lo que las cosas son en sí mismas. De allí se seguiría que el sentido interno —en virtud de su dependencia material del sentido externo- tampoco nos podría otorgar un conocimiento de las cosas en sí mismas, sino que este será un conocimiento que refleja las relaciones entre los objetos y el sujeto. Por otro lado, Kant introduce un segundo argumento en virtud del cual está vedado el acceso a la cosa en sí. Según este argumento, el tiempo contiene en sí mismo, con independencia de la experiencia efectiva, relaciones de sucesión, simultaneidad y permanencia. Todos los fenómenos serán puestos en la mente humana bajo alguna de estas relaciones, las cuales no dependen de la experiencia, sino que son su condición de posibilidad. Dado que estas relaciones temporales poseen su origen a priori en el sujeto, se sigue que el sentido interno sólo puede brindarnos un conocimiento acerca del modo en el cual nosotros colocamos las representaciones en nuestra mente. $\mathrm{Ni}$ la sucesión, ni la simultaneidad, ni la permanencia pueden ser atribuidas a las cosas en sí mismas, sino que dependen necesariamente del modo en el cual nuestra subjetividad organiza las representaciones de los fenómenos y es consciente de ellas ${ }^{3}$.

En este punto, Kant comienza a introducir el problema de la auto-afección en los siguientes términos:

[...] aquello que, como representación, puede preceder a toda acción de pensar algo cualquiera, es la intuición, y si ella no contiene nada más que relaciones, [es] la forma de la intuición, la cual, como no representa nada, salvo en la medida en que algo es puesto en la mente, no puede ser otra cosa que la manera como la mente es

2. Cfr. KANT, I. Crítica de la razón pura, pp. 116-117.

3. Kant menciona aquí tres modos de la temporalidad: sucesión, simultaneidad y permanencia. Considero que estas relaciones suponen la aplicación de las categorías al múltiple sensible y constituyen, de ese modo, determinaciones objetivas del tiempo. Por el contrario, cuando la intuición interna o sentido interno es identificado con la forma de la sucesión, nos hallamos ante un tiempo meramente subjetivo que describe el flujo de representaciones que se da en nuestra mente, haciendo abstracción de la aplicación de las categorías. 
afectada por su propia actividad, a saber, por este poner las representaciones de ella, ${ }^{4}$ y por tanto, por sí misma, es decir, [no puede ser otra cosa que] un sentido interno, según su forma. ${ }^{5}$

Aquella representación que puede preceder al pensamiento de algo es la intuición. En el marco del criticismo kantiano, esta precedencia no debe ser interpretada en términos temporales, sino en sentido lógico, como aquella representación, presupuesta en la aplicación de las categorías. En la medida en que tal representación intuitiva sólo contenga relaciones, nos hallamos ante la forma de la intuición. Ya hemos visto que el contenido material de nuestra intuición interna coincide con el contenido material de las representaciones de los sentidos externos. Pero lo que ahora estamos analizando no es el contenido material de la intuición, sino su forma. Ésta no puede tener su origen en la experiencia, pues nos hallamos ante un tipo de intuición que posibilita toda experiencia y que Kant denomina forma de la intuición ${ }^{6}$. Kant sostiene que la forma de la intuición no puede tener un origen empírico ${ }^{7}$, puesto que se trata de una forma que hace posible

4. Es decir, de la intuición. Ed. Acad. interpreta $<<$ de la mente $>>$, modificando el texto [...] (Nota del traductor).

5. KANT, I. Crítica de la razón pura, pp. 116-117.

6. La forma de la intuición se refiere a la forma en la cual es recibida el múltiple sensible, mientras que intuición formal remite al hecho de que espacio y tiempo son ellos mismos intuiciones que poseen unidad y dentro de los cuales es posible la recepción del múltiple sensible. La forma de la intuición presupone la intuición formal, pues de otro modo tal forma carecería de unidad y nos hallaríamos ante una multiplicidad de tiempos y espacios. Cfr. DÜSING, K. Objektive und subjektive Zeit. Untersuchungen zu Kants Zeittheorie und zu ihrer modernen kritischen Rezeption. Kant-Studien, Berlin/New York, v. 71, 1980. pp. 1-34 (aquí, p. 9) y NAKANO, H. Selbstaffektion in der transzendentalen Deduktion. Kant-Studien, Berlin/New York, vol. 102 n 2, 2011. pp. 213-231 (aquí, p. 217).

7. El origen causal de la afección subyacente a toda constitución de objetos representa un gran problema dentro del marco del criticismo kantiano. Si consideramos que el objeto que nos afecta es una cosa en sí, estaríamos estableciendo una relación causal entre la cosa en sí y nuestra capacidad representativa - lo cual constituye una aplicación de la categoría de causalidad, que excede los límites de nuestro conocimiento posible-. Y si consideramos que la causa de la afección es un fenómeno, caemos en una suerte de circularidad según la cual la materia de los fenómenos posee un origen causal 
la experiencia misma. El único origen posible de esta representación proviene de una auto-afección. Se trata de un acto mediante el cual la mente es afectada por su propia actividad de poner en sí misma ciertas representaciones. Es decir, la mente se auto-afecta al poner en sí misma la forma de la intuición, colocando las representaciones empíricas en un orden temporal determinado ${ }^{8}$. Esta forma de la intuición es un sentido interno, mediante el cual la mente se percata de su propia actividad.

A continuación, Kant subraya el carácter fenoménico del propio yo que aparece ante sí mismo. Dado que el yo —i.e. la actividad de la propia mente- sólo aparece ante sí mismo mediante la forma de la intuición o el sentido interno, este yo no se muestra tal como es en sí mismo, sino que aparece condicionado por la forma de la intuición. Kant está rechazando la posibilidad de que el yo se intuya a sí mismo mediante una intuición intelectual — la cual sería el acto de la mera espontaneidad y no necesitaría del recurso a la sensibilidad-. Sólo podemos tener una conciencia intuitiva de nuestro yo, en la medida en que éste se afecte a sí mismo y aparezca de manera sensible, estableciendo así la forma de la intuición que precede a toda experiencia posible. En la medida en que el yo se afecta a sí mismo, aparece ante sí como un fenómeno, cuya forma es la forma de la intuición. Cabe destacar que asistimos aquí a un desdoblamiento de la subjetividad, en la cual el yo afectante y el yo que aparece no coinciden, pues en un caso se trata de una mera forma lógica que debe poder acompañar a nuestras representaciones, mientras que en el segundo caso se trata de un flujo de representaciones que aparecen sucesivamente. Sin embargo, podemos sostener que el yo fenoménico supone la auto-afección —es decir, el influjo del yo trascendental sobre la propia

fenoménico. Cfr. JÁUREGUI, C. Sentido interno y subjetividad: un análisis del problema del auto-conocimiento en la filosofía trascendental de Kant. Buenos Aires: Prometeo, 2008, p. 73.

8. Vemos aquí la presencia de dos niveles: 1) la forma de la intuición, la cual es una forma a priori i 2) las representaciones empíricas que son colocadas en un orden temporal acorde a esa forma a priori. Esto nos permite anticipar la distinción de dos sentidos de auto-afección: uno a priori y otro empírico. Una interpretación análoga es desarrollada por Zoeller. Cfr. ZOELLER, G. Making Sense out of inner Sense: The Kantian Doctrine as Illuminated by the Leningrad Reflexion. International Pbilosopbical Quarterly, New York, v. XXIX, n. 3, 1989. pp. 263-270. 
mente- - sin que ello signifique que el yo fenoménico es el aparecer del yo trascendental—el cual jamás puede aparecer de manera sensible-.

Con el problema de la auto-afección se plantea la siguiente pregunta: ¿cómo es posible que el sujeto aparezca de manera intuitiva y por tanto sensible, no como un objeto de los sentidos externos - es decir a través de un cuerpo- sino como un objeto del sentido interno? Quedan así señalados dos aspectos que debe cumplir ese yo del cual se debe poder ser consciente gracias a la auto-afección. Primero, se trata de un aparecer intuitivo y no meramente intelectual. Por ello no basta con la simple representación intelectual Yo, pues ésta es un mero pensamiento que en sí mismo no implica ninguna multiplicidad sensible que pueda ser recibida por el sujeto y de la cual podamos tener una intuición. En segundo lugar, se trata de una intuición interior de sí mismo y no exterior — tal como la que estaría implicada con el aparecer de nuestro cuerpo como algo que se mueve en el espacio y ocupa un lugar en él一. Así pues, Kant reformula en los siguientes términos el problema de la auto-afección:

Si la facultad de hacerse consciente de sí mismo ha de recoger (aprehender) lo que yace en la mente, esta [facultad] debe afectarla a ella, y sólo de esa manera [esa facultad] puede producir una intuición de sí misma, cuya forma empero [...] determina, en la representación del tiempo, la manera como lo múltiple está reunido en la mente; porque ella se intuye a sí misma, no como se representaría a sí misma inmediatamente de manera espontánea, sino según la manera como es afectada por dentro, y en consecuencia, como se aparece a sí misma, [y] no como es. ${ }^{9}$

La facultad de bacerse consciente de sí mismo se refiere a la representación simple del Yo, la cual es un producto de la espontaneidad de nuestro pensamiento. Se trata del Yo de la apercepción trascendental. Este yo (trascendental) sólo ha de aprehender la multiplicidad que yace en la mente, bajo la condición de que él afecte a la mente. Nótese que Kant nos habla aquí de un aprebender lo que yace en la mente. Como es sabido, en el marco del criticismo kantiano, la aprehensión es un acto que recorre el múltiple sensible de manera sucesiva y lo reúne en una representación unitaria a fin de que el múltiple pueda ser reconocido

9. KANT, I. Crítica de la razón pura, pp. 117-118. 
como tal. En efecto, si el múltiple no fuese reunido, nos hallaríamos ante representaciones inconexas que en rigor no compondrían una multiplicidad $^{10}$. La auto-afección consiste en el acto de afectar la mente, aprehendiendo de manera sucesiva un múltiple sensible dado y reuniéndolo, a fin de reconocerlo como tal. La mente recibe intuitivamente un dato sensible, pero el mismo es ordenado de manera sucesiva, sólo en virtud de un influjo proveniente del yo activo que las aprehende unas tras otras ${ }^{11}$.

Así pues, la auto-afección posee al menos tres funciones. La primera consiste en organizar los datos sensibles bajo la forma de la sucesión, pues sólo de esa forma pueden ser aprehendidos. La segun$\mathrm{da}$, íntimamente ligada a la primera, consiste en afectar la mente al establecer la forma a priori —a saber: la sucesión — bajo la cual el múltiple sensible se organiza en nuestra mente. La tercera implica un aparecer fenoménico del propio yo ante sí mismo, el cual sólo puede aparecer de manera sucesiva - es decir, como un flujo de representaciones - puesto que esa es la forma a priori de nuestra mente, que ha sido generada por el mismo acto de auto-afección ${ }^{12}$. No se trata aquí de sostener que la sucesión es una forma originaria de nuestro yo activo - pues eso generaría un problema al interior de la teoría al reducir la forma de la sensibilidad a una forma del entendimiento-, sino de subrayar que sólo podemos ser conscientes de la sucesión gracias a este influjo proveniente del yo activo, sin el cual la sucesión no sería reconocida como tal.

Como veremos a continuación, la doctrina de la auto-afección se despliega en dos niveles: uno se refiere al aparecer de representa-

10. Cfr. KANT, I. Crítica de la razón pura, p. 170 y ss.

11. Moreau sostiene que la sucesión mediante la cual aprehendemos las representaciones no puede ser dada de manera pasiva, sino que es el producto de la reflexión sobre la síntesis de una multiplicidad exterior, la cual si bien es dada pasivamente de manera simultánea, sólo puede ser aprehendida de manera sucesiva. Cfr. MOUREAU, J. Intuition et Appréhension. Kant-Studien, Berlin/New York, v. 71, n. 3, 1980. pp. 282-298 (aquí, p. 291 y ss).

12. Klemme sostiene que la noción de auto-afección subraya el carácter fenoménico del yo que aparece ante nuestro sentido interno, evitando así una posible confusión entre lo nouménico y lo fenoménico en el aparecer intuitivo del yo ante sí mismo. Cfr. KLEMME, H. Kants Pbilosopbie des Subjekts: Systematische und entwicklungsgescbicbtliche Untersucbungen zum Verbältnis von Selbstbewußtsein und Selbsterkenntnis. Hamburg: Felix Meiner Verlag, 1996, p. 229. 
ciones empíricas ante el sentido interno y el otro se identifica con la toma de consciencia explícita cuyo objeto temático es la forma de la intuición que se encuentra a la base de toda experiencia posible ${ }^{13}$.

\section{La auto-afección en el $§ 24$ de la Crítica de la razón pura.}

El segundo texto que nos interesa se halla en el § 24 de la primera Crítica. Allí Kant se refiere a la paradoja del sentido interno, la cual consiste en la incapacidad de acceder a lo que nuestro yo es en sí mismo. Se trata de una tesis paradójica en la medida en que aquello que nos es más íntimo - a saber, nuestra propia subjetividad— sólo puede ser intuido adoptando, al igual que los objetos del sentido externo, un carácter fenoménico ${ }^{14}$. Se vuelve a subrayar la tesis ya mencionada en el § 8, según la cual está vedada la presentación intuitiva de lo que es nuestro yo en sí mismo. En el $§ 24$ aparece esbozado cierto desdoblamiento de nuestro yo, pues un aspecto de nosotros debe comportarse de manera pasiva a fin de recibir la afección del propio yo en tanto facultad activa ${ }^{15}$. Por ello, Kant sostiene la distin-

13. Jáuregui considera que la auto-afección implica un cambio de perspectiva que considera las representaciones como estados de conciencia que ya no hace presente los objetos en el espacio. Lo que la mente pone en sí misma a través de la auto-afección se halla en un tiempo subjetivo. Cfr. JÁUREGUI, C. Sentido interno y subjetividad: un análisis del problema del auto-conocimiento en la filosofía trascendental de Kant. p. 81. Esta lectura se halla en consonancia con la distinción efectuada por Kemp Smith entre el objeto de la representación - el cual supone la afección externa-y la representación como estado interno del yo. Cfr. KEMP SMITH, N. A Commentary to Kant's Critique of Pure Reason. Cuarta edición. London: Palgrave MacMillan, 2003, p. 294. Creo que estas lecturas deben ser complementadas con la interpretación de G. Zoeller, quien sostiene que además de la "auto-afección empírica" hay una "auto-afección a priori" mediante la cual se genera la forma de la intuición a partir de la síntesis figurativa de la imaginación tal como aparece en el § 24 de la Crítica de la razón pura. Cfr. ZOELLER, G. Making Sense out of inner Sense: The Kantian Doctrine as Illuminated by the Leningrad Reflexion, p. 263-270.

14. También es paradójico el acto mediante el cual se nos presenta el contenido del sentido interno - a saber: el acto de auto-afección-. Cfr. JÁUREGUI, C. Sentido interno y subjetividad: un análisis del problema del auto-conocimiento en la filosofía trascendental de Kant, p. 75.

15. El yo es doblemente pasivo y doblemente activo. Por un lado, es pasivo con relación a la afección externa y en el vínculo con la afección interna. Por 
ción cuidadosa entre sentido interno y apercepción. Mientras que el primero consiste en cierta forma pasiva que es afectada, el segundo de estos términos se refiere al yo afectante, que determina nuestro sentido interno: ${ }^{16}$

El entendimiento [...] bajo la denominación de síntesis trascendental de la imaginación, ejerce sobre el sujeto pasivo cuya facultad es él [mismo], aquella acción de la cual decimos, correctamente, que por ella es afectado el sentido interno. ${ }^{17}$

Con la denominación de sintesis trascendental de la imaginación se traza una distinción entre el enlace meramente intelectual, pensado en la unidad de las categorías y aquel enlace que ya no es meramente intelectual, sino que remite al enlace de lo múltiple de la intuición sensible humana ${ }^{18}$. En este contexto, la imaginación es definida por Kant como "[...] la facultad de representar en la intuición un objeto aun sin la presencia de el". ${ }^{19}$ Es la síntesis trascendental de la imaginación, la que por un lado permite que nos representemos un objeto en la intuición. La síntesis trascendental de la imaginación es la que me permite, por ejemplo, imaginar el lado no visto de un objeto o proyectar un momento pasado y un momento futuro que se conectan con el

otro lado, es activo con la multiplicidad proveniente del sentido externo y con aquélla que se presenta meramente ante el sentido interno. Cfr. JÁUREGUI, C. Sentido interno y subjetividad: un análisis del problema del auto-conocimiento en la filosofía trascendental de Kant, p. 81.

16. Según Nakano, no se trata de la presencia de dos sujetos numéricamente distintos y existentes por fuera de la relación. Se trata más bien de dos aspectos de una misma actividad, pues el sentido interno no es otra cosa más que ser afectado por el entendimiento. Cfr. NAKANO, H. Selbstaffektion in der transzendentalen Deduktion. Kant-Studien, Berlin/New York, v. 102, n. 2, 2011, pp. 213-231 (aquí, p. 228).

17. KANT, I. Crítica de la razón pura, p. 219.

18. Según Baum la distinción en dos partes de la "Deducción trascendental" en la segunda edición de la Crítica de la razón pura, se basa en la distinción entre síntesis intelectual y síntesis figurativa. Cfr. BAUM, M. Deduktion und Beweis in Kants Transzendentalpbilosopbie: Untersucbungen zur Kritik der reinen Vernunft. Königstein: Hain Verlag bei Athenäum, 1986, pp. 79-81 y BAUM, M. The B-Deduction and the Refutation of Idealism. The Southern Journal of Pbilosopby, Memphis, v. XXV, Suplement, 1986, pp. 89-107 (aquí, pp. 102-104).

19. KANT, I. Crítica de la razón pura, p. 117-118. 
presente en el cual me encuentro. Por ello, se trata de una síntesis que permite enlazar el múltiple sensible —incluso aquél que se encuentra ausente- al configurar de manera originaria las formas puras del espacio y el tiempo. En la medida en que se trata de una facultad que remite a la intuición, la imaginación pertenece a la sensibilidad. Pero el enlace efectuado por esta síntesis de la imaginación es llevado a cabo de acuerdo con la unidad de nuestro yo activo — que se identifica con el yo pienso de la apercepción trascendental—. En esta medida, se trata de una facultad que pertenece a nuestra espontaneidad. Kant no se refiere aquí a una imaginación empírica que reproduce siguiendo pasivamente las leyes de asociación y supone una experiencia ya constituida, sino que se trata de una facultad, cuyo rasgo esencial es la espontaneidad que se halla a la base de la experiencia misma. La imaginación empírica o reproductiva es aquella que actúa, por ejemplo, al vincular el verano con el calor o el cielo estrellado con la noche. Kant la llama imaginación reproductiva, para distinguirla así de la imaginación productiva que se halla sometida a la unidad de nuestro entendimiento puro y permite efectuar la síntesis figurativa. Como se observa en el pasaje que estamos analizando, el entendimiento afecta nuestro sentido interno, a través de la síntesis trascendental de la imaginación. Es decir, la sintesis figurativa se identifica con la auto-afección. ${ }^{20}$

Así pues, debemos distinguir tres niveles íntimamente relacionados. En primer término, encontramos un sujeto activo que se identifica con la unidad sintética de la apercepción. Se trata de la fuente originaria de todo enlace que "[...] se dirige al múltiple de las intuiciones en general $;[\mathrm{y}]$ con el nombre de las categorías, [se dirige, $]$ antes de toda intuición sensible, a objetos en general $[\ldots]^{\prime \prime}{ }^{21}$ En segundo lugar, hallamos un sentido interno que "[...] contiene la mera forma de la intuición, pero sin enlace de lo múltiple en ella, y por tanto, no con-

20. Al interpretar el rol de la imaginación como una mera relación entre el entendimiento y la sensibilidad, tomo distancia de la interpretación efectuada por Heidegger, quien habría sostenido la primacía de la imaginación en relación con las otras facultades y habría realizado una suerte de sustancialización de éstas. Cfr. HEIDEGGER, M. Kant und das Problem der Metapbysik. Frankfurt am Main: Klostermann, 1991. pp. 138-141, 164 y NAKANO, H. Selbstaffektion in der transzendentalen Deduktion, p. 228.

21. KANT, I. Crítica de la razón pura, p. 220. 
tiene todavía ninguna intuición determinada $[\ldots]^{\prime \prime}{ }^{22}$ Por un lado, vemos un sujeto activo que se dirige a objetos en general - antes $^{23}$ de toda intuición sensible-. Por otro lado, hallamos un sentido interno, cuyo múltiple sensible carece de enlace y determinación. A fin de que nuestra espontaneidad se dirija efectivamente a la intuición sensible y esta última adquiera cierta determinación debemos considerar un tercer nivel de la subjetividad, a saber: la determinación de nuestro sentido interno "[... ] por medio de la acción trascendental de la imaginación (influjo sintético del entendimiento sobre el sentido interno), a la que llamé síntesis figurativa" ${ }^{\prime 24}$. La auto-afección consiste en un acto por medio del cual el múltiple que aparece ante nuestro sentido interno adquiere determinación al ser enlazado por medio de la síntesis trascendental de la imaginación. ${ }^{25} \mathrm{El}$ múltiple sensible no existe en tanto tal antes de la auto-afección, sino que el sólo es posible una vez que fue enlazado mediante la síntesis figurativa que permite su organización bajo la forma de la intuición. Es decir, la sucesión o forma del sentido interno es un resultado del influjo de nuestra espontaneidad sobre

22. Idem, ibidem.

23. En rigor, no existe este sujeto activo que prescinde de la intuición sensible, pues para nosotros los humanos, el yo pienso sólo es tal en la medida en que se dirige a un múltiple sensible. Sin embargo, como resultado del análisis, Kant postula este sujeto activo que se dirige a una intuición en general -haciendo abstracción de la intuición sensible que de hecho poseemos.

24. KANT, I. Crítica de la razón pura, p. 220.

25. Se ha señalado que la auto-afección no presupone un múltiple dado de antemano que deba ser sintetizado, sino que se trata del vínculo originario entre el entendimiento y el sentido interno - vínculo que sería efectuado por la síntesis trascendental de la imaginación. Según esta lectura, la recepción del múltiple sensible sólo es posible gracias a la unidad formal de nuestro sentido interno, la cual es deudora de un acto de auto-afección. Cfr. NAKANO, H. Selbstaffektion in der transzendentalen Deduktion, pp. 213 231. Otros intérpretes sostienen que la auto-afección presupone un múltiple dado de antemano, el cual aparece bajo la forma de la intuición (Form der Anschauung) pero sin unidad - es decir, sin que se halle constituida la intuición formal (formelle Anschaunng), la cual sería un producto de la auto-afección. Cfr. MOHR, G. Das sinnliche Icb: inneren Sinn und Bewußtsein bei Kant. Würzburg: Königshausen \& Neumann, 1991, pp. 109-121; y STRAWSON, P. The Bounds of Sense. London: Metheuen \& Co. Ltd., 1966, p. 32. Considero que esta última línea interpretativa difiere con lo que efectivamente sostiene Kant. 
nuestro sentido interno, por intermedio de la síntesis trascendental de la imaginación.

Kant introduce algunos ejemplos que permitirían comprender en qué consiste la auto-afección. Por ejemplo, sólo podemos representarnos en el pensamiento una línea, al trazarla en el pensamiento, de acuerdo con el concepto de lo que es una línea. El múltiple sensible que el sujeto recibe y que carece de enlace, es enlazado de manera originaria por un acto del entendimiento, mediado por la imaginación productiva. Nuestro sentido interno adquiere de ese modo determinación, pues ahora contiene la representación de una línea y no meramente un múltiple carente de unidad. Kant agrega unas líneas más adelante una reflexión en torno a la representación del tiempo:

[...] tampoco [podemos representarnos] el tiempo, sin prestar atención, en el trazado de una línea recta (destinada a ser la representación figurativa externa del tiempo), meramente a la acción de síntesis de lo múltiple, por la cual determinamos sucesivamente el sentido interno, $\mathrm{y}$ [sin prestar atención] por ese medio a la sucesión de esta determinación en él. ${ }^{26}$

No significa que el acto de trazar una línea en nuestro pensamiento se halle a la base de toda experiencia, sino de un acto que se encuentra a la base de aquel particular tipo de experiencia que podemos llegar a tener de nosotros mismos cuando intentamos hacer intuitiva nuestra propia espontaneidad. Si queremos ser conscientes acerca de los caracteres esenciales de aquella intuición pura que precede a priori a toda intuición y de la cual sólo podemos ser concientes mediante el influjo de nuestra espontaneidad sobre nosotros mismos, debemos representarnos el trazado de una recta y particularmente, debemos prestar atención a la síntesis sucesiva de las múltiples partes que componen la recta. A medida que avanzamos en el trazado de la recta, debemos retener los elementos de la línea ya trazados y enlazar a ellos las nuevas partes de la recta. De ese modo, lo múltiple es enlazado de manera sucesiva y por ello el trazado de la línea recta constituye la representación figurativa de la forma sucesiva de nuestro sentido interno. Además, el acto de trazar la línea nos permite intuir el movimiento de nuestra subjetividad, aquel acto espontáneo gracias al cual enlazamos un múltiple sensible. El acto de atención aquí menciona-

26. KANT, I. Crítica de la razón pura, p. 220. 
do podría ser interpretado como un cambio de perspectiva, mediante el cual dirigimos nuestra mirada a la forma sucesiva en la que aprehendemos el múltiple sensible. Es decir, sólo tenemos una consciencia intuitiva de nuestro yo, si desplazamos la atención hacia nuestra actividad trascendental y tratamos de obtener una representación de ella - hemos visto que sólo podemos obtener una representación figurativa de aquella forma de la intuición que posibilita nuestra experiencia-. Sólo si prestamos atención a la síntesis de lo múltiple implicada en el trazado de una línea recta, podemos ser conscientes de un modo reflexivo de la sucesión. Kant agrega que el concepto mismo de sucesión es un producto de la auto-afección:

El movimiento, como acción del sujeto (no como determinación de un objeto), y en consecuencia, la síntesis de lo múltiple en el espacio, cuando hacemos abstracción de éste y sólo atendemos a la acción por la cual determinamos el sentido interno de acuerdo con la forma de él, es incluso lo que produce, ante todo, el concepto de la sucesión. [...] no es que el entendimiento encuentre ya en éste un enlace tal de lo múltiple, sino que lo produce, al afectarlo. ${ }^{27}$

En primer lugar, se sostiene que el múltiple sintetizado es un múltiple espacial. Es decir, el contenido material de nuestro sentido interno coincide con el de nuestro sentido externo. En segundo término, podríamos hablar un cambio de perspectiva, en virtud del cual ya no consideramos a las representaciones en la medida en que remiten a objetos en el espacio, sino que hacemos abstracción de tal referencia objetiva y tratamos de atender al acto mediante el cual determinamos nuestro sentido interno, es decir, reflexionamos acerca de nuestra aprehensión sucesiva de representaciones que, si bien remiten a objetos espaciales, aquí son consideradas como meros estados de conciencia que se ordenan sucesivamente ${ }^{28} .{ }^{29}$ Finalmente, queda destacada la tesis según la cual esta forma sucesiva del sentido interno no

27. Idem, p. 220-221.

28. Cfr. JÁUREGUI, C. Sentido interno y subjetividad: un análisis del problema del auto-conocimiento en la filosofía trascendental de Kant, p. 87.

29. La determinación sintética de lo múltiple espacial y la posición de ese múltiple en nuestro sentido interno son dos momentos de la auto-afección que sólo pueden ser separados por abstracción. Cfr. DIRSCHAUER, S. La théorie kantienne de l'auto-affection. Kant-Studien, Berlin/New York, v. 95, n. 1, 2004, pp. 53-85 (aquí, p. 75). 
es dada de manera pasiva sino que es un producto de la auto-afección, es decir, del influjo del entendimiento sobre nuestro sentido interno ${ }^{30}$. Esta determinación del sentido interno puede ser analizada en dos niveles: por un lado, en un nivel a priori la auto-afección produce de manera originaria la forma de la intuición bajo la cual se organiza el múltiple de nuestro sentido interno; por otro lado, en un nivel empírico, gracias a la auto-afección las representaciones de objetos espaciales pasan a ser consideradas como meros estados de conciencia, estableciendo de ese modo una determinación material de nuestro sentido interno.

Por último, hallamos una nota introducida por Kant en la cual queda sugerida la auto-afección, concebida ya no en un nivel a priori - es decir, como una toma de conciencia de la forma de la intuiciónsino en un nivel empírico, mediante el cual determinaríamos de manera contingente nuestro sentido interno:

No se ve cómo se puede encontrar tanta dificultad en que el sentido interno sea afectado por nosotros mismos. Todo acto de atención puede darnos un ejemplo de ello. El entendimiento determina siempre, en él, al sentido interno de acuerdo con el enlace que él piensa, de modo que llegue a producirse una intuición interna que corresponda al múltiple en la síntesis del entendimiento. En qué medida es afectada con ello habitualmente la mente, podrá percibirlo cada cual en sí mismo. ${ }^{31}$

El sentido interno es determinado por el entendimiento en todo acto de atención. ${ }^{32}$ Es decir, toda síntesis efectuada por el entendimiento puede producir una intuición interna que se corresponde con el múl-

30. La auto-afección, al ser tematizada como aquel acto del entendimiento que le otorga unidad a nuestro sentido interno, muestra que receptividad y espontaneidad son aspectos inseparables. Cfr. NAKANO, H. Selbstaffektion in der transzendentalen Deduktion, p. 230.

31. KANT, I. Crítica de la razón pura, p. 222.

32. Algunos comentaristas ven en el acto de atención la auto-afección propiamente dicha y no un mero ejemplo empírico. Cfr: ZÖELLER, G. Making Sense out of inner Sense: The Kantian Doctrine as Illuminated by the Leningrad Reflexion, pp. 267-270; ALLISON, H. Kant's Transcendental Idealism: an Interpretation and Defense. New Heaven/London: Yale University Press, 1983, p. 268; DÜSING, K. Objektive und subjektive Zeit. Untersuchungen zu Kants Zeittheorie und zu ihrer modernen kritischen Rezeption, p. 24. 
tiple que fue enlazado. ${ }^{33}$ Esto significa que es la misma representación empírica la que se constituye como un fenómeno que se halla o bien en el tiempo objetivo, o bien en lo que podríamos denominar tiempo meramente subjetivo. Este cambio de perspectiva es posibilitado por un acto de atención mediante el cual ponemos de relieve ante nuestra conciencia el hecho de que poseemos una representación o estado de consciencia y dejamos en suspenso la inserción de esta representación en el tiempo objetivo.

\section{La auto-afección y el esquematismo trascendental: tiempo subjetivo y tiempo objetivo.}

La determinación a priori del sentido interno que da lugar a la forma sucesiva de nuestra aprehensión no implica necesariamente la validez objetiva de tal determinación temporal. En la "Segunda Analogía de la Experiencia" Kant sostiene, a modo de ejemplo, que si bien percibimos las diferentes partes de una casa de manera sucesiva, sabemos que objetivamente las partes se hallan en relaciones temporales de simultaneidad. ${ }^{34}$ Es decir, la doctrina de la auto-afección da lugar a la consciencia de un tiempo meramente subjetivo, que si bien es $a$ priori, no garantiza la validez objetiva de tales determinaciones temporales. A fin de comprender esta distinción entre una temporalidad subjetiva y otra objetiva, podríamos contrastar en sus rasgos esencia-

33. Düsing sostiene que el acto de atención subraya que en la auto-afección las representaciones oscuras que se encuentran dadas en el sentido interno son llevadas a la claridad y a la distinción respecto de otras representaciones del sentido interno, constituyendo así una determinación del tiempo subjetivo como forma de nuestro sentido interno. Cfr. DÜSING, K. Objektive und subjektive Zeit. Untersuchungen zu Kants Zeittheorie und zu ihrer modernen kritischen Rezeption, p. 24 y ss. Allison sostiene que mediante la auto-afección hacemos de nuestras representaciones "objetos subjetivos" al reflexionar sobre nuestros estados de conciencia. Cfr. ALLISON, H. Kant's Transcendental Idealism: an Interpretation and Defense, p. 261.

34. La distinción entre tiempo objetivo y tiempo subjetivo se halla en: KANT, I. Crítica de la razón pura, pp. 283 y ss.; 292 y ss.; 300. Düsing sostiene que la vivencia subjetiva del tiempo y la determinación de los fenómenos reales son a menudo incongruentes. Si bien las determinaciones del tiempo subjetivo son $a$ priori, ello no garantiza la validez objetiva de tales determinaciones. Cfr. DÜSING, K. Objektive und subjektive Zeit. Untersuchungen zu Kants Zeittheorie und zu ihrer modernen kritischen Rezeption, pp. 10 y ss. 
les algunos aspectos de la doctrina de la auto-afección y el esquematismo trascendental.

La función de los esquemas trascendentales es salvar la radical heterogeneidad entre los conceptos puros del entendimiento y las intuiciones sensibles, para hacer posible la aplicación de las categorías a la sensibilidad. Los esquemas trascendentales son un producto de la imaginación trascendental. Estos son homogéneos con las categorías, pero al mismo tiempo son sensibles, motivo por el cual pueden trazar una suerte de puente entre el entendimiento y la sensibilidad ${ }^{35}$. Considero que si bien tanto en el esquematismo como en la auto-afección que da lugar a la forma de la intuición nos hallamos ante una determinación del sentido interno, efectuada por la imaginación trascendental, se trata de determinaciones que poseen diferentes funciones. Así pues, mientras que la función de los esquemas trascendentales consiste en posibilitar la aplicación de las categorías, es decir, en hacer posible la subsunción de intuiciones bajo los conceptos puros del entendimiento, la función de la forma de la intuición consiste en hacer posible la receptividad del múltiple sensible que es aprehendido sucesivamente. Si bien la forma de la intuición - y la síntesis figurativa que la hace posible - se hallan orientadas, en principio, a la constitución de la objetividad, el rasgo específico de la auto-afección involucra un cambio de perspectiva que posibilita el aparecer intuitivo de la propia subjetividad. Mediante la auto-afección a priori el sujeto estaría tomando consciencia de un modo explícito del carácter subjetivo de la forma de la intuición. Un segundo aspecto que nos permite distinguir la doctrina de la auto-afección del esquematismo trascendental es el siguiente: la forma de la intuición es una forma de la sensibilidad — no del entendimiento- aún cuando su unidad sea deudora de la espontaneidad del sujeto. Por el contrario, los esquemas

35. Esta interpretación del esquematismo se opone a aquella línea que identifica a estos últimos con una regla del entendimiento. Dentro de esta última línea de investigación podemos mencionar a los siguientes autores: ARENAS, L. El esquematismo de los conceptos matemáticos: una interpretación. Endoxa, Madrid, n. 8, 1997, pp. 111-136; BUTTS, R. Kant's Schemata as Semantical Rules. In: BECK LEWIS, W. Kant Studies Today. Illinois: Open Court, 1969, pp. 290-300; SEEL, G. Die Einleitung in die Analytik der Grundsätze, der Schematismus und die obersten Grundsätze (A 130/B 169 - A 158/B 197). In: MOHR, G. y WILLASCHEK, M (Hrg). Immanuel Kant. Kritik der reinen Vernunft. Berlin: Akademie Verlag, 1998, pp. 217-246. 
trascendentales son representaciones puras que no pertenecen exclusivamente a la sensibilidad, sino que poseen una parte intelectual y otra parte sensible — constituyéndose, de ese modo, en el elemento mediador entre la sensibilidad y el entendimiento- ${ }^{36}$. Así por ejemplo, Kant introduce el esquema correspondiente al concepto de causa en los siguientes términos:

El esquema de la causa y de la causalidad de una cosa en general es lo real, a lo cual, cuando es puesto, siempre le sigue algo diferente. Consiste, pues, en la sucesión de lo múltiple, en la medida en que está sometida a una regla. ${ }^{37}$

En primer lugar, podemos comparar el esquema y la forma de la intuición, tomando como criterio la función que ambos desempeñan. En la sucesión que se identifica con la forma de la intuición, si bien el sentido interno es determinado por la actividad del entendimiento, tal modo de la sucesión no tiene como finalidad garantizar la subsunción de intuiciones empíricas bajo categorías, sino dar cuenta del aparecer intuitivo del yo. Por el contrario, la sucesión de lo múltiple como esquema de la causalidad tiene como función otorgar un criterio universal que garantice la subsunción de los fenómenos bajo la categoría de causalidad. ${ }^{38}$

Contrastemos ahora el carácter mixto del esquema con el carácter puramente sensible de la forma de la intuición. El esquematismo nos presenta una noción de sucesión que se halla necesariamente sometida a una regla — a saber: la regla de causalidad-y en esa presencia de la regla radica el aspecto intelectual del esquema trascendental, mientras que la parte sensible se halla en lo múltiple que se da sucesivamente. Por el contrario, la sucesión que resulta de la auto-afección

36. Cfr. MOLEDO, F. Función sistemática y naturaleza del esquematismo trascendental. Ágora: Papeles de Filosofía, Santiago de Compostela, v. 30, n. 2, 2011, pp. 163-185; LA ROCCA, C. Schematismus und Anwendung. Kant-Studien, Berlin/New York, v. 80, n. 2, 1989, pp. 139-154.

37. KANT, I. Crítica de la razón pura, p. 243.

38. Philonenko interpreta el esquematismo trascendental como un principio metódico que le otorga significado a las categorías. Tal significado no es sino el objeto empírico particular. Cfr. PHILONENKO, A. Lectura del esquematismo trascendental. Ágora: Papeles de Filosofía, Santiago de Compostela, v. 7 n. 1, 1988, pp. 9-25. 
es una forma pura de nuestra sensibilidad, sin explicar nada acerca de nuestro entendimiento.

No se trata tanto de subrayar la existencia de dos flujos de consciencia - numéricamente distintos e independientes-sino más bien, de un flujo de consciencia que adopta la forma de la sucesión y puede ser analizado desde diversas perspectivas. Una de ellas subraya el principio metódico que posibilita la subsunción de intuiciones empíricas bajo las categorías y alude a un tipo de temporalidad que está compuesta por un aspecto sensible y uno intelectual. La otra perspectiva se identifica con la forma de la intuición interna, pero hace abstracción de la referencia a objetos, para prestar atención al aparecer intuitivo del sujeto. La determinación del sentido interno - en el contexto de la auto-afección — supone la constitución de objetos - y por tanto, el esquematismo trascendental—. Pero la sucesión meramente subjetiva que se produce gracias a la auto-afección, sólo permite intuir el propio yo, sin que ello garantice la validez objetiva de lo que nos representamos. ${ }^{39}$ La regla que opera en el marco de la auto-afección va acompañada de una suerte de abstracción o puesta en suspenso de la referencia objetiva, dejando a la sucesión del sentido interno como una forma puramente sensible que carece de la legalidad que posee el esquema. Así pues, la sucesión como forma de la intuición interna implica un mero fluir de estados de consciencia, sin que ello se corresponda con el tiempo de los estados objetivos ${ }^{40}$. A diferencia de ello, la sucesión como esquema trascendental de la categoría de causalidad hace referencia a un modo de organización puro de las representaciones, consideradas como objetos y no como meros estados subjetivos, pues sólo de ese modo la sucesión de lo real — como esquema de la causalidad - estaría operando como elemento mediador entre las intuiciones empíricas y los conceptos puros.

\section{Conclusiones}

Se ha señalado que el modo de temporalidad del cual somos conscientes gracias a la auto-afección puede ser pensado como un

39. Cfr. JÁUREGUI, C. Sentido interno y subjetividad: un análisis del problema del auto-conocimiento en la filosofía trascendental de Kant, p. 85.

40. Cfr. MOREAU, J. Intuition et Appréhension. p. 296. 
tiempo subjetivo que se distingue de las determinaciones temporales objetivas. Este acto de auto-afección puede ser analizado en dos niveles. La auto-afección a priori, consiste en la explícita toma de consciencia de aquella forma de la intuición que surge como resultado de la síntesis figurativa. En cambio, la auto-afección empírica es la toma de consciencia reflexiva de las representaciones empíricas y contingentes que se despliegan ante nuestro sentido interno, considerando a las representaciones como meros estados mentales. En ambos casos, se supone la actividad sintética del entendimiento y la constitución de la objetividad, así como el esquematismo trascendental que garantiza la subsunción de intuiciones empíricas bajo conceptos puros. Esto es así puesto que aquello que afecta al sujeto no es sino la actividad del entendimiento en su enlace de un múltiple sensible que hace posible la experiencia. Sin embargo, hemos dado razones para sostener que el modo de temporalidad que se identifica con la forma del sentido interno debe ser distinguido de las determinaciones temporales objetivas que sólo serían posibles en virtud de los esquemas trascendentales.

\section{Referências}

ALLISON, H. Kant's Transcendental Idealism: an Interpretation and Defense. New Heaven/London: Yale University Press, 1983.

ARENAS, L. El esquematismo de los conceptos matemáticos: una interpretación. Endoxa, Madrid, n. 8, 1997, pp. 111-136.

BAUM, M. Deduktion und Beweis in Kants Transzendentalpbilosopbie: Untersucbungen zur Kritik der reinen Vernunft. Königstein: Hain Verlag bei Athenäum, 1986.

The B-Deduction and the Refutation of Idealism. The Southern Journal of Pbilosopby, Memphis, v. XXV, Suplement, 1986, pp. 89-107.

BUTTS, R. Kant's Schemata as Semantical Rules. In: BECK LEWIS, W. Kant Studies Today. Illinois: Open Court, 1969, pp. 290-300.

DIRSCHAUER, S. La théorie kantienne de l'auto-affection. Kant-Studien, Berlin/New York, v. 95, n. 1, 2004, pp. 53-85.

DÜSING, K. Objektive und subjektive Zeit. Untersuchungen zu Kants Zeittheorie und $\mathrm{zu}$ ihrer modernen kritischen Rezeption. KantStudien, Berlin/New York, v. 71, 1980, pp.1-34.

HEIDEGGER, M. Kant und das Problem der Metapbysik. Frankfurt am Main: Klostermann, 1991. 
JÁUREGUI, C. Sentido interno y subjetividad: un análisis del problema del auto-conocimiento en la filosofía trascendental de Kant. Buenos Aires: Prometeo, 2008.

KANT, I. Kants Werke. Akademie Textausgabe. Berlin: Walter de Gruyter, 1968.

Crítica de la razón pura. Traducción de Mario Caimi. Buenos Aires: Colihue, 2007.

KEMP SMITH, N. A Commentary to Kant's Critique of Pure Reason. Cuarta edición. London: Palgrave MacMillan, 2003.

KLEMME, H. Kants Pbilosophie des Subjekts: Systematische und entwicklungsgeschicbtliche Untersucbungen zum Verbältnis von Selbstbewußtsein und Selbsterkenntnis. Hamburg: Felix Meiner Verlag, 1996.

LA ROCCA, C. Schematismus und Anwendung. Kant-Studien, Berlin/ New York, v. 80, n. 2, 1989, pp. 139-154.

MOHR, G. Das sinnliche Icb: inneren Sinn und Bewußtsein bei Kant. Würzburg: Königshausen \& Neumann, 1991.

Moledo, F. Función sistemática y naturaleza del esquematismo trascendental. Ágora: Papeles de Filosofía, Santiago de Compostela, v. 30, n. 2, 2011, pp. 163-185.

MOREAU, J. Intuition et Appréhension. Kant-Studien, Berlin/New York, v. 71 n. 3, 1980, pp. 282-298.

NAKANO, H. Selbstaffektion in der transzendentalen Deduktion. KantStudien, Berlin/New York, v. 102, n. 2, 2011, pp. 213-231.

PHILONENKO, A. Lectura del esquematismo trascendental. Ágora: Papeles de Filosofía, Santiago de Compostela, v. 7, n. 1, 1988, pp. 9-25.

SEEL, G. Die Einleitung in die Analytik der Grundsätze, der Schematismus und die obersten Grundsätze (A 130/B 169 - A 158/B 197). In: MOHR, G. y WILLASCHEK, M (Hrg). Immanuel Kant. Kritik der reinen Vernunft. Berlin: Akademie Verlag, 1998, pp. 217-246.

STRAWSON, P. The Bounds of Sense. London: Metheuen \& Co. Ltd., 1966.

ZOELLER, G. Making Sense out of inner Sense: The Kantian Doctrine as Illuminated by the Leningrad Reflexion. International Pbilosopbical Quarterly, New York, v. XXIX, n. 3, 1989, pp. 263-270.

Recebido em 21.01.2014

Aceito em 18.03.2014 